\title{
Estimating Parameters of Optimal Average and Adaptive Wiener Filters for Image Restoration with Sequential Gaussian Simulation
}

Tuan D Pham

\section{Linköping University Post Print}

\section{Tweet}

N.B.: When citing this work, cite the original article.

(C)2016 IEEE. Personal use of this material is permitted. However, permission to reprint/republish this material for advertising or promotional purposes or for creating new collective works for resale or redistribution to servers or lists, or to reuse any copyrighted component of this work in other works must be obtained from the IEEE.

Tuan D Pham , Estimating Parameters of Optimal Average and Adaptive Wiener Filters for Image Restoration with Sequential Gaussian Simulation, 2015, IEEE Signal Processing Letters, (22), 11, 1950-1954.

http://dx.doi.org/10.1109/LSP.2015.2448732

Postprint available at: Linköping University Electronic Press

http://urn.kb.se/resolve?urn=urn:nbn:se:liu:diva-128596 


\title{
Estimating Parameters of Optimal Average and Adaptive Wiener Filters for Image Restoration with Sequential Gaussian Simulation
}

\author{
Tuan D. Pham, Senior Member, IEEE
}

\begin{abstract}
Filtering additive white Gaussian noise in images using the best linear unbiased estimator (BLUE) is technically sound in a sense that it is an optimal average filter derived from the statistical estimation theory. The BLUE filter mask has the theoretical advantage in that its shape and its size are formulated in terms of the image signals and associated noise components. However, like many other noise filtering problems, prior knowledge about the additive noise needs to be available, which is often obtained using training data. This paper presents the sequential Gaussian simulation in geostatistics for measuring signal and noise variances in images without the need of training data for the BLUE filter implementation. The simulated signal variance and the BLUE average can be further used as parameters of the adaptive Wiener filter for image restoration.
\end{abstract}

Index Terms - Image restoration, best linear unbiased estimator, optimal average filter, adaptive Wiener filter, sequential Gaussian simulation, kriging.

\section{BACKGROUND}

Image restoration is the process of recovering the original image from its degraded version, which is subject to the corruption of noise. The reduction of various types of noise in images has been an active area of research in image processing and computer vision. In particular, additive Gaussian noise is the most common noise source, as its behavior and effect are resembled by many random processes that occur in nature. In addition, Gaussian noise models have been frequently used and addressed in various applications, because of its mathematical tractability in both spatial and frequency domains [1].

While there are many methods developed for the removal of additive random noise in images, which are selectively found in [2]-[9], this paper focuses on the estimation of the parameters for the optimal best linear unbiased estimator (BLUE) average and adaptive Wiener filters. In fact, the Wiener filter and its modified versions have been found useful to the processing of advanced biological and medical image signals [10], [11]. Thus, a brief background about the degradation model as well as the popular adaptive Wiener filter are presented first as follows.

A digital image degraded with additive random noise can be modeled as [12]

Tuan D. Pham is with the Aizu Research Cluster for Medical Engineering and Informatics, Center for Advanced Information Science and Technology, The University of Aizu, Aizuwakamatsu, Japan. E-mail: tdphameu-aizu.ac.jp

Copyright (c) 2015 IEEE. Personal use of this material is permitted. However, permission to use this material for any other purposes must be obtained from the IEEE by sending an email to pubs-permissions@ieee.org.

$$
y_{j}=f_{j}+\nu_{j},
$$

where $y_{j}$ is the degraded digital image, $f_{j}$ is the original digital image, and $\nu_{j}$ represents the signal-independent additive random noise.

Furthermore, if $\nu_{j}$ is zero mean and white with variance $\sigma_{\nu}^{2}$, and $f_{j}$ is assumed to be stationary and within a small local region, then $f_{j}$ can be modeled as [13], [14]

$$
f_{j}=m_{f}+\sigma_{f} b_{j},
$$

where $m_{f}$ and $\sigma_{f}$ are the local mean and standard deviation of $f_{j}$, respectively; and $b_{j}$ is the zero-mean white noise variable with unit variance. The above equation models $f_{j}$ as a sum of a space-variant local mean $m_{f}$ and white noise with spacevariant local variance $\sigma_{f}^{2}$.

The Wiener filter provides the restored image $f_{j}^{W}$ within the local region by [12]

$$
f_{j}^{W}=m_{f}+\frac{\sigma_{f}^{2}}{\sigma_{f}^{2}+\sigma_{v}^{2}}\left[\left(y_{f}-m_{f}\right)\right] .
$$

The adaptive Wiener filter attempts to suppress noise in a digital image using $m_{f}$ and $\sigma_{f}^{2}$ that are updated at each pixel $j$ as follows [12]:

$$
f_{j}^{W}=m_{f}(j)+\frac{\sigma_{f}^{2}(j)}{\sigma_{f}^{2}(j)+\sigma_{v}^{2}}\left[y_{j}-m_{f}(j)\right],
$$

in which if the noise variance is not known, the adaptive Wiener filter calculates $\sigma_{v}^{2}$ as the average of all the estimated local variances.

The next section presents the derivation of an optimal average image filter using the BLUE.

\section{BLUE-BASED IMAGE FILTER}

Based on the noise model outlined earlier, Equation (2) can be rewritten as

$$
f_{j}=m_{f}+v_{j}, j=1, \ldots, J
$$

where $v_{j}=\sigma_{f} b_{j}$, and $J$ are the number of local pixels.

An optimal estimate of $m_{f}$, denoted as $\hat{m}_{f}$, can be formulated as a weighted linear combination of the local pixels:

$$
\hat{m}_{f}=\sum_{j=1}^{J} w_{j} f_{j}
$$


Assuming that the expected values of the $v_{j}$ are $E\left(v_{j}\right)=0$, and the $v_{j}$ are uncorrelated, giving $E\left(v_{j} v_{k}\right)=0$, for $j \neq k$, and the variances of the $v_{j}$ are $E\left(v_{j}^{2}\right)=\sigma_{j}^{2}>0$. The estimate $\hat{m}_{f}$ is unbiased if $E\left(\hat{m}_{f}\right)=m_{f}$, resulting in $\sum_{j=1}^{J} w_{j} E\left(m_{f}\right)=$ $E\left(m_{f}\right)$, which restricts $\sum_{j=1}^{J} w_{j}=1$ [15], [16]. Furthermore, the best linear unbiased estimator (BLUE) means that $E\left[\hat{m}_{f}-\right.$ $\left.\left.m_{f}\right)^{2}\right]$ is to be minimized, or the $w_{j}$ must minimize

$$
E\left(\sum_{j=1}^{J} \sum_{k=1}^{J} w_{j} w_{k} v_{j} v_{k}\right)=\sum_{j=1}^{J} w_{j}^{2} \sigma_{j}^{2},
$$

subject to

$$
\sum_{j=1}^{J} w_{j}=1
$$

By rewriting Equation (8) as

$$
1=\sum_{j=1}^{J} w_{j}=\sum_{j=1}^{J}\left(w_{j} \sigma_{j}\right) \frac{1}{\sigma_{j}} .
$$

This minimization problem can be solved without calculus by using the Cauchy's Inequality [15] that gives

$$
1 \leq \sqrt{\sum_{j=1}^{J} w_{j}^{2} \sigma_{j}^{2}} \sqrt{\sum_{j=1}^{J} \frac{1}{\sigma_{j}^{2}}}
$$

with equality if and only if

$$
w_{j} \sigma_{j}=\beta \frac{1}{\sigma_{j}},
$$

where $\beta$ is a constant. Thus,

$$
w_{j}=\beta \frac{1}{\sigma_{j}^{2}} .
$$

Summing on both sides of Equation (12) and using Equation (8) gives

$$
\beta=\left(\sum_{j=1}^{J} \frac{1}{\sigma_{j}^{2}}\right)^{-1} .
$$

The BLUE for $f_{i}$ can now be obtained as

$$
\hat{m}_{f}=\beta \sum_{j=1}^{J} \frac{f_{j}}{\sigma_{j}^{2}} .
$$

If all the $\sigma_{j}^{2}$ are the same, the BLUE for $m_{f}$ becomes the arithmetic mean of all $f_{j}$. Equation (14) yields the same filter mask $\frac{\beta}{\sigma_{j}^{2}}$ using the Bayesian BLUE as described in [17].

The next section presents the notion of sequential Gaussian simulation in geostatistics, which can be applied to estimate $\sigma_{j}^{2}$ required for the calculation of $\hat{m}_{f}$ expressed in Equation (14). In turn, the noise variance $\sigma_{v}^{2}$ specified in Equation (4) can be computed as the average of all the simulated local variances $\sigma_{j}^{2}$. Thus, two pixel-wise updated parameters $m_{f}(j)$ and $\sigma_{f}^{2}(j)$, and the constant noise variance $\sigma_{v}^{2}$ can be estimated for the performance of the adaptive Wiener filter.

\section{Estimating Filter Parameters With Sequential GAUSSIAN SIMULATION}

Sequential Gaussian simulation (SGS) is a stochastic method for generating partial realizations using multivariate normal random functions, and kriging estimator in geostatistics [18]. The basic notion of SGS is established on the following theorem that proves the equivalence between drawing from a multivariate distribution and from a sequence of univariate distributions conditional to univariate realizations. Let $Z(x)$ be a subset of $N$ variables of a random function, and $z(x)$ be a sampling of size $n$. The conditional cummulative frequency distribution function $F\left(x_{1}, x_{2}, \ldots, x_{N} ; t_{1}, t_{2}, \ldots, t_{N} \mid n\right)$ is given by

$$
\begin{aligned}
& F\left(x_{1}, x_{2}, \ldots, x_{N} ; t_{1}, t_{2}, \ldots, t_{N} \mid n\right)= \\
& F\left(x_{1} ; t_{1} \mid n\right) F\left(x_{2} ; t_{2} \mid n+1\right) \ldots F\left(x_{n} ; t_{n} \mid n+N-1\right)
\end{aligned}
$$

whose proof can be constructed using Bayes' theorem, and provided in [18].

Another important theorem for SGS states that if a kriging error for the kriged estimate $Z^{K}\left(x_{i}\right)$ of the sample at location $i$ is normally distributed with zero mean and variance: $N\left(0, \sigma^{2}\left(x_{i}\right)\right)$, then the probability distribution for the true value is $N\left(\hat{z}\left(x_{i}\right), \sigma^{2}\left(x_{i}\right)\right)$ (also see [18] for its proof).

The procedure of SGS starts with the concept of kriging [18], [16], [19]. The kriging estimate of $f_{i}$, denoted as $f_{i}^{K}$, is computed as

$$
f_{i}^{K}=\sum_{j=1}^{n} a_{j} f_{j}
$$

where $f_{j}$ is the known intensity value of the pixel at location $j, n$ is the number of neighbors of the pixel whose value is to be estimated, and $a_{j}$ are the kriging weights to be determined by solving the following ordinary kriging system [16]:

$$
\sum_{j=1}^{n} a_{j} \gamma(k j)-\lambda=\gamma(k i), \forall k=1, \ldots, n
$$

where $k j=|k-j|=h$, and

$$
\sum_{j=1}^{n} a_{j}=1 .
$$

The variance of the kriging estimation error is given by [16]

$$
\sigma_{K}^{2}\left(f_{i}\right)=\sum_{j=1}^{n} a_{j} \gamma(j i)+\lambda .
$$

The semi-variogram, $\gamma(h)$, of an image is defined as the half of the average squared difference between the paired pixel intensities $(k i)$ of which distance is separated by a lag or Euclidean distance $h$ [16]:

$$
\gamma(h)=\frac{1}{2 N(h)} \sum_{(k i),|k-i|=h}\left(f_{k}-f_{i}\right)^{2}
$$




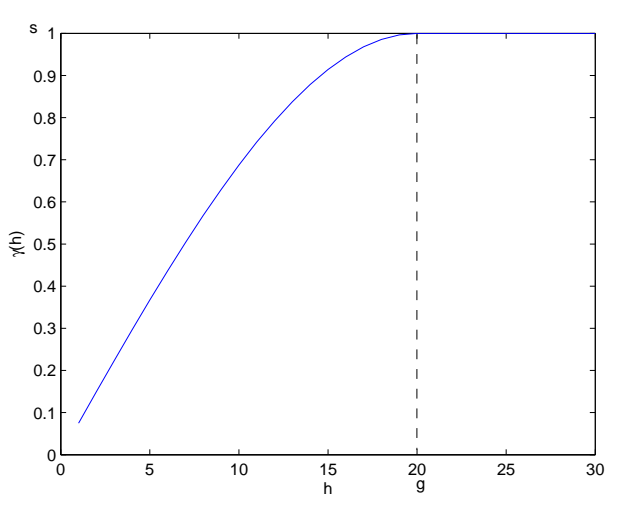

Fig. 1. Theoretical semi-variogram using the spherical model with $s=1$ and $g=20$.

TABLE I

PSNR (DB) OF RESTORATION RESULTS OF THE LENA IMAGE DEGRADED WITH DIFFERENT NOISE LEVELS OF $N\left(\mu, \sigma^{2}\right)$.

\begin{tabular}{lcccc}
\hline Noise level & AF & AWF & SOAF & SAWF \\
\hline$N(0,0.001)$ & 32.69 & 34.44 & 32.89 & 36.10 \\
$N(0,0.01)$ & 30.70 & 31.06 & 30.71 & 33.33 \\
$N(0,0.05)$ & 29.08 & 29.07 & 29.27 & 31.60 \\
$N(0,0.1)$ & 28.56 & 28.52 & 28.64 & 30.78 \\
\hline
\end{tabular}

where $N(h)$ is the number of pairs of $f_{k}$ and $f_{i}$ that are separated by lag $h$.

The function defined in Equation (20) is called the experimental semi-variogram. The experimental semi-variogram is considered isotropic when it depends only on the lag $h$, and anisotropic when it varies in different directions. Thus, the experimental semi-variogram must be prepared for different directions given the configuration of the data. In this study, $h$ is taken in both horizontal and vertical directions of the image.

The theoretical semi-variogram is a function represented by a model equation. A widely used theoretical semi-variogram is the spherical or the Matheron model, which is used in this study and defined as [16]

$$
\gamma(h)= \begin{cases}s\left[1.5 \frac{h}{g}-0.5\left(\frac{h}{g}\right)^{3}\right] & : \quad h \leq g \\ s & : h>g\end{cases}
$$

where $g$ and $s$ are called the range and the sill of the theoretical semi-variogram, respectively; which can be estimated using the experimental semi-variogram.

Figure 1 shows the spherical semi-variogram model defined in (21). When $h=0$, two samples are taken at the same position, and the difference between the two must be zero. When $h>0$, the two samples move a distance apart and some positive difference between the two values can be expected. As the samples move further apart, the differences should increase accordingly. Ideally when the distance becomes very large and reaches $g$, the sample values become independent of one another. The semi-variance $\gamma(h)$ will then become constant at $s$ as the result of the calculation of the difference between the pairs of independent samples.
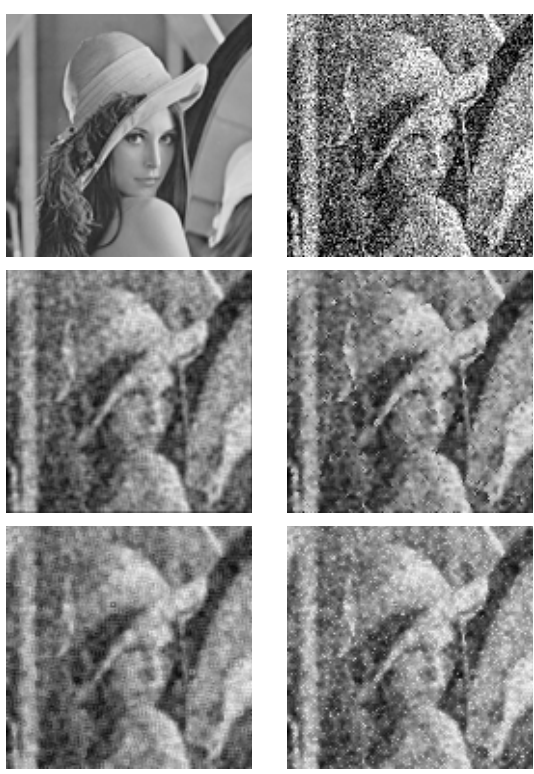

Fig. 2. Lena image: original (top-left), degraded with $N(0,0.05)$ (top-right), average filter (middle-left), adaptive Wiener filter (middle-right), simulationbased optimal average filter (bottom-left), and simulation-based adaptive Wiener filter (bottom-right)
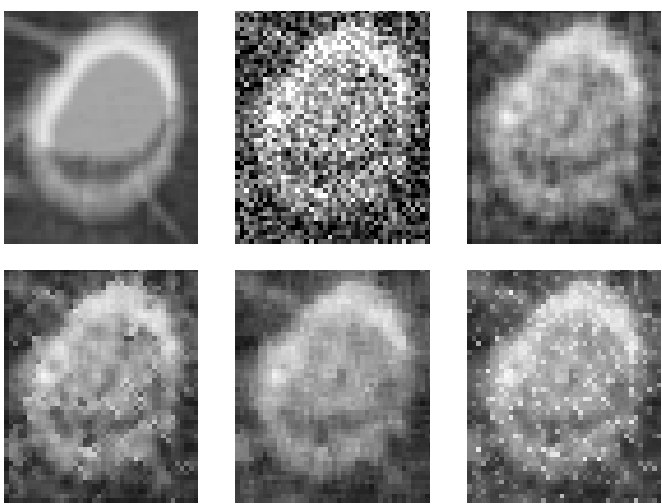

Fig. 3. PET-CT image of a lung tumor: original (top-left), degraded with $N(0,0.05)$ (top-middle), average filter (top-right), adaptive Wiener filter (bottom-left), simulation-based optimal average filter (bottom-middle), and simulation-based adaptive Wiener filter (bottom-right).
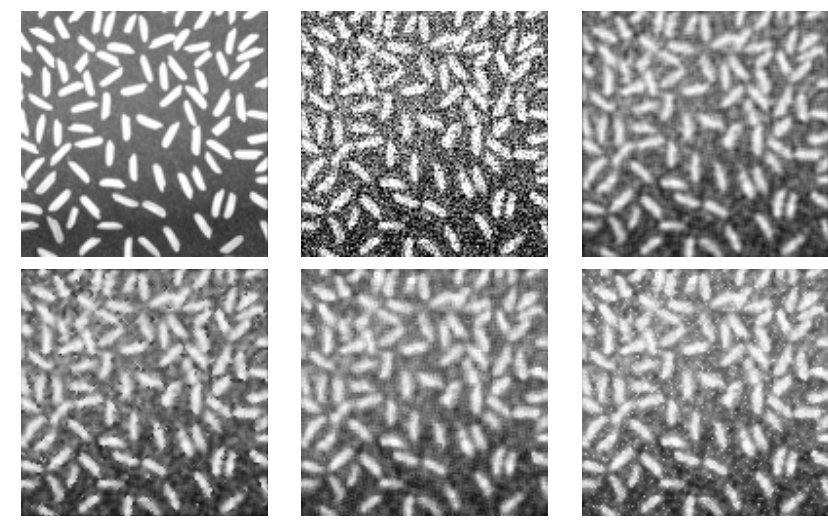

Fig. 4. Image of rice grains: original (top-left), degraded with $N(0,0.05)$ (top-middle), average filter (top-right), adaptive Wiener filter (bottom-left), simulation-based optimal average filter (bottom-middle), and simulation-based adaptive Wiener filter (bottom-right). 
TABLE II

PSNR (DB) OF RESTORATION RESULTS OF THE PET-CT IMAGE OF A LUNG TUMOR DEGRADED WITH DIFFERENT NOISE LEVELS OF $N\left(\mu, \sigma^{2}\right)$.

\begin{tabular}{lcccc}
\hline Noise level & AF & AWF & SOAF & SAWF \\
\hline$N(0,0.001)$ & 32.91 & 35.61 & 34.43 & 38.69 \\
$N(0,0.01)$ & 30.81 & 31.10 & 31.95 & 34.39 \\
$N(0,0.05)$ & 28.91 & 28.81 & 29.51 & 31.57 \\
$N(0,0.1)$ & 28.61 & 28.48 & 29.22 & 31.26 \\
\hline
\end{tabular}

TABLE III

PSNR (DB) OF RESTORATION RESULTS OF THE IMAGE OF RICE GRAINS DEGRADED WITH DIFFERENT NOISE LEVELS OF $N\left(\mu, \sigma^{2}\right)$.

\begin{tabular}{lcccc}
\hline Noise level & AF & AWF & SOAF & SAWF \\
\hline$N(0,0.001)$ & 30.21 & 31.21 & 30.26 & 31.93 \\
$N(0,0.01)$ & 29.04 & 29.33 & 29.19 & 30.82 \\
$N(0,0.05)$ & 28.03 & 27.84 & 28.41 & 29.75 \\
$N(0,0.1)$ & 27.85 & 27.59 & 28.12 & 29.40 \\
\hline
\end{tabular}

Based on the two theorems and the method of kriging presented above, the algorithm for SGS is described as follows [18], [19].

\section{Algorithm for SGS with Image Data}

1) Transform image data to standard normal distribution if the sampling is not univariate normal.

2) Use the experimental semi-variogram to construct a suitable theoretical semi-variogram for the transformed data.

3) Select randomly a pixel at location $i$, which is without a value and due for the generation of a simulated value, and perform kriging to obtain the estimate of $f_{i}$ (Equation (16)) by means of the image intensities of its neighboring pixels, and then the corresponding kriging variance (Equation (19)) by means of the theoretical semi-variogram.

4) Draw a random residual $r_{i}$ that follows a normal distribution $N\left(f_{i}^{K}, \sigma_{K}^{2}\left(f_{i}\right)\right)$

5) The simulated value is the sum of the kriged estimate and residual: $f_{i}^{s}=f_{i}^{K}+r_{i}$.

6) Add $f_{i}^{s}$ to the set of the image data.

7) If $f_{i}$ is not the last pixel without a value, go to Step 2.

8) If Step 1 was performed, back transform the values in the multivariate realization to the original space.

By performing a number of simulations on an image, $\sigma_{j}^{2}$ expressed in Equation (14) can be statistically obtained, which leads to the estimation of adaptive Wiener filter parameters $m_{f}(j), \sigma_{f}^{2}(j)$, and $\sigma_{v}^{2}$, required for computing Equation (4).

\section{EXPERIMENT}

The proposed approach for estimating the parameters of the optimal average and adaptive Wiener filter was tested using the Lena image of $128 \times 128$ pixels, a PET-CT image of a lung tumor of $51 \times 43$ pixels, and an image of rice grains of $128 \times 128$ pixels. The three original images were degraded with different levels of white Gaussian noise distribution of zero mean $(\mu=0)$ and variance $\sigma^{2}$. Each of the degraded images was used to perform 10 sequential Gaussian simulations of $70 \%$ of the image data, using the public-domain BMELIB software [20], which also automatically estimated the sill and range for the theoretical variogram using the information provided by the experimental one. The image signal and noise variances were computed using the simulated images. The image signal variances were used for the processing of the optimal average filter. The image signal and noise variances obtained from the simulated images together with the local intensity mean values obtained from the optimal average filter were then used for the processing of the adaptive Wiener filter.

The peak signal-to-noise ratio (PSNR) expressed in $\mathrm{dB}$ was used to compare the performance of the average filter (AF), adaptive Wiener filter (AWF), simulation-based optimal average filter (SOAF), and simulation-based adaptive Wiener filter (SAWF). A higher PSNR generally indicates that the image restoration is of higher quality. The PSNR is defined as [21]:

$$
\mathrm{PSNR}=10 \log _{10}\left(\frac{f_{\max }^{2}}{\mathrm{MSE}}\right),
$$

where $f_{\max }$ is taken as the maximum value of the image data type, which is 255 for the intensity range $[0,255]$ used in this paper, and MSE is the mean square error between the original image $f$ and processed image $\hat{f}$ of $M \times N$ size:

$$
\operatorname{MSE}=\frac{1}{M N} \sum_{j=1}^{M N}\left(f_{j}-\hat{f}_{j}\right)^{2} .
$$

Using a filter mask of $3 \times 3$, the PSNR of the three images obtained for the AF, AWF, SOAF, and SAWF are given in Tables I-III. Figures 2-4 show the original Lena, tumor, and rice images, in which the original images were degraded with white Gaussian noise of $\mu=0$ and $\sigma^{2}=0.05$, and images restored by the AF, AWF, SOAF, and SAWF, respectively. In particular, although with some visible noise highlights, the bottom-right image of Figure 3, which was processed by SAWF, was restored with the desirable high-detail image region of the tumor, showing glucose (sugar) solution that contains a very small amount of radioactive material absorbed by the tissues.

The SAWF achieves the highest PSNR in all noise levels of all three images, in which the PSNR improvements for the tumor image are the highest. The PSNR values of the SOAF are higher than those of the AF in all noise levels of all three images, and the AWF in the cases of the images being degraded with higher noise levels $(N(0,0.05)$ and $N(0,0.1))$. The experimental results consistently show the noice-reduction improvements of the simulation-based optimal average filter over the average filter, and the simulation-based adaptive Wiener filter over the adaptive Wiener filter.

\section{CONCLUSION}

The sequential Gaussian simulation has been utilized for estimating the parameters of the optimal average and adaptive Wiener filters without the requirement of training data. The proposed approach can be extended using multivariate kriging [22] to perform the restoration of noisy color images. 


\section{REFERENCES}

[1] R.C. Gonzalez, R.E. Woods, Digital Image Processing, 3rd edition. New Jersey: Prentice-Hall, 2008.

[2] E. Luo, S.H. Chan, T.Q. Nguyen, "Adaptive image denoising by targeted databases", IEEE Trans Image Processing, vol. 24, pp. 2167-2181, 2015.

[3] K.M. Mohamed, R.C. Hardie, "A collaborative adaptive Wiener filter for image restoration using a spatial-domain multi-patch correlation model", EURASIP J. Advances in Signal Processing, vol. 2015:6, 2015. doi: 10.1186/s13634-014-0189-3

[4] H. Talebi, P. Milanfar, "Global image denoising", IEEE Trans Image Processing, vol. 23, pp. 755-768, 2014.

[5] W. Liu, W. Lin, "Additive white Gaussian noise level estimation in SVD domain for images", IEEE Trans Image Proc, vol. 22, pp. 872-883, 2013.

[6] F. Luisier, T. Blu, M. Unser, "Image denoising in mixed PoissonGaussian noise", IEEE Trans Image Processing, vol. 20, pp. 696-708, 2011.

[7] Y. Xiao, T. Zeng, J. Yu, M.K. Ng, "Restoration of images corrupted by mixed Gaussian-impulse noise via $l_{1}-l_{0}$ minimization", Pattern Recognition, vol. 44, pp. 1708-1720, 2011.

[8] P. Gravel, G. Beaudoin, J.A. De Guise, "A method for modeling noise in medical images", IEEE Trans Med Imaging, vol. 23, pp. 1221-1232, 2004.

[9] T.D. Pham, “An image restoration by fusion", Pattern Recognition, vol. 34, pp. 2403-2411, 2001.

[10] C.V. Cannistraci, A. Abbas, X. Gao, "Median modified Wiener filter for nonlinear adaptive spatial denoising of protein NMR multidimensional spectra”, Scientific Reports, vol. 5, 8017, 2015. doi:10.1038/srep08017
[11] P.F. Nunes, M.L.N. Franco, J.B.D. Filho, A.C. Patrocnio, " Intensity transform and Wiener filter in measurement of blood flow in arteriography," Proc. SPIE Medical Imaging, vol. 9413, 941326, 2015. doi: $10.1117 / 12.2082419$

[12] J.S. Lim, Two-Dimensional Signal and Image Processing. New Jersey: Prentice Hall, 1990.

[13] H.J. Trussell, B.R. Hunt, "Sectioned methods for image restoration", IEEE Trans Acoust, Speech, Signal Proc, vol. 26, pp. 157-164, 1978.

[14] D.T. Kuan, A.A. Sawchuk, T.C. Strand, P. Chavel, "Adaptive noise smoothing filter for images with signal-dependent noise," IEEE Trans Pattern Anal Mach Intell., pp. 165-177, 1985.

[15] C.L. Byrne, The First Course in Optimization. Boca Raton: CRC Press, 2015.

[16] E.H. Isaaks, R.M. Srivastava, An Introduction to Applied Geostatistics. New York: Oxford University Press, 1989.

[17] K. Krajsek, R. Mester, H. Scharr, "Statistically optimal averaging for image restoration and optical flow estimation", DAGM 2008, LNCS, vol. 5096, pp. 466-475, 2008.

[18] R.A. Olea, Geostatistics for Engineers and Earth Scientists. Boston: Kluwer Academic Publishers, 1999.

[19] C.V. Deutsch, Geostatistical Reservoir Modeling. New York: Oxford University Press, 2002.

[20] G. Christakos, P. Bogaert, M.L. Serre, Temporal GIS: Advanced Functions for Field-Based Applications. New York: Springer-Verlag, 2002.

[21] A.R. Weeks, Fundamentals of Electronic Image Processing. Washington: SPIE, New York: IEEE Press, 1996.

[22] H. Wackernagel, Multivariate Geostatistics: An Introduction with Applications, 3rd edition. Berlin: Springer-Verlag, 2003. 